\title{
In vivo multiscale photoacoustic microscopy of human skin
}

Christopher P. Favazza, Song Hu, Victor Huang, Omar Jassim, Lynn A. Cornelius, et al.

Christopher P. Favazza, Song Hu, Victor Huang, Omar Jassim, Lynn A. Cornelius, Lihong V. Wang, "In vivo multiscale photoacoustic microscopy of human skin," Proc. SPIE 7899, Photons Plus Ultrasound: Imaging and Sensing 2011, 789946 (22 February 2011); doi: 10.1117/12.875969 


\title{
In Vivo Multi-scale Photoacoustic Microscopy of Human Skin
}

\author{
Christopher. P. Favazza*a ${ }^{\mathrm{a}}$, Song $\mathrm{Hu}^{{ }^{\mathrm{a}}}$, Victor Huang ${ }^{\mathrm{b}}$, Omar Jassim ${ }^{\mathrm{b}}$, Lynn A. Cornelius ${ }^{\mathrm{b}}$, and \\ Lihong V. Wang ${ }^{\text {ta }}$ \\ ${ }^{a}$ Optical Imaging Laboratory, Department of Biomedical Engineering, Washington University in St. \\ Louis, 1 Brookings Drive, St. Louis, MO, USA 63130; \\ ${ }^{b}$ Division of Dermatology, Washington University School of Medicine, 660 S. Euclid Ave. \\ St. Louis, MO, USA 63110
}

\begin{abstract}
Scalability is a key feature of photoacoustic microscopy (PAM). Reports have shown that PAM systems can be designed to possess sub-micron resolution at shallow depths or penetrate centimeters deep at the expense of resolution while the number of resolved pixels in the depth direction remains high. This capability to readily tune the imaging parameters while maintaining the same inherent contrast could be extremely useful for a variety of biomedical applications. Human skin, with its layered vascular structure whose dimensions scale with depth, provides an ideal imaging target to illustrate this advantage. Here, we present results from in vivo human skin imaging experiments using two different PAM systems, an approach which enables better characterization of the cutaneous microvasculature throughout the imaging depth. Specifically, we show images from several distinct areas of skin: the palm and the forearm. For each region, the same area was imaged with both an optical-resolution PAM (OR-PAM) and an acoustic-resolution PAM (AR-PAM), and the subsequent images were combined into composite images. The OR-PAM provides less than $5 \mu \mathrm{m}$ lateral resolution, capable of imaging the smallest capillary vessels, while the AR-PAM enables imaging at depths of several millimeters. Several structures are identifiable in the ORPAM images which cannot be differentiated in AR-PAM images, namely thin epidermal and stratum corneum layers, undulations in the dermal papillae, and capillary loops. However, the AR-PAM provides images of larger vessels, deeper than the OR-PAM can penetrate. These results demonstrate how PAM's scalability can be utilized to more fully characterize cutaneous vasculature, potentially impacting the assessment of numerous cardiovascular related and cutaneous diseases.
\end{abstract}

Keywords: photoacoustic microscopy, multi-scale, skin, microvasculature, optical resolution, acoustic resolution

*equal contribution authors

† lhwang@biomed.wustl.edu, http://oilab.seas.wustl.edu

\section{INTRODUCTION}

Photoacoustic microscopy (PAM) is inherently scalable. A PAM system can be designed to possess optically limited lateral resolution (OR-PAM). In this case sub-micron later resolution is possible; however, at limited imaging depths, i.e. within the quasi-ballistic photo regime $(\sim 1 \mathrm{~mm}$ in tissue)[1]. Further a PAM can be designed to possess acoustically limited later resolution, and can provide high resolution images with optical absorption contrast at extraordinary depths, reaching the hard optical penetration limit (more than several $\mathrm{cm}$ in tissue)[2].

Human skin offers an exceptional target to illustrate the scalability of PAM. Particularly, the size of vessels comprising cutaneous vascular networks scale with depth [3,4]. Capillaries populate the superficial layers of the skin and are well within the quasi-ballistic photon regime, whereas larger vessels are found deeper beneath the surface. With the ability to better and more thoroughly characterize cutaneous circulation, PAM could impact diagnosis and treatment of circulatory[5] and dermatological[6] diseases as well as foster better understanding of drug delivery and uptake through the skin[4].

Here we demonstrate PAM's unique capability to scale imaging parameters appropriate for a desired target. In a series of imaging experiments, a volunteer's palm and forearm were imaged with both an OR-PAM and an AR-PAM. The same

Photons Plus Ultrasound: Imaging and Sensing 2011, edited by Alexander A. Oraevsky, Lihong V. Wang, Proc. of SPIE Vol. 7899,789946 - @ 2011 SPIE · CCC code: 1605-7422/11/\$18 · doi: $10.1117 / 12.875969$ 
area in each region of the skin was imaged and the data was co-registered, showing the complementary structures imaged by both systems.

\section{METHODS}

\subsection{Systems descriptions}

Using an AR-PAM[7, 8] and an OR-PAM[1], we imaged cutaneous microvasculatures in skin from the forearm and the palm. The AR-PAM is connected to a laser system consisting of an Nd:YLF pump laser and dye laser. The output beam from the dye laser is coupled into a multi-mode fiber and routed to the scan head of the microscope. Part of the beam exiting the fiber is scavenged and directed to a photodiode to record pulse to pulse energy variations. The rest of the beam passes through a conical lens, forming a ring-shaped or donut pattern. The beam passes around the ultrasonic transducer and is then directed towards the sample through a weakly focusing optical condenser. The weakly focused laser beam is confocally and coaxially aligned with the acoustic focus of the ultrasound transducer. Custom written data acquisition and motor control software synchronize the motor position, laser firing events, and data acquisition. As the sample is raster scanned, all data is collected, digitized, and recorded onto a PC (Figure 1). The OR-PAM is connected to a high repetition rated Nd:YAG laser. High laser repetition rates are necessary for fast scanning, which is extremely important for human imaging. Similar to the AR-APM, the laser beam is coupled into a single mode fiber and routed to the scan head of the microscope. Again, a portion of the beam is gleaned and directed to a photodiode for pulse to pulse laser energy calibration. The laser beam is tightly focused by an objective lens with an NA of 0.1 . The focused beam passes through an optical-acoustic coupler and illuminates the target, as shown in Figure 1. Again, the optical and acoustic foci are confocally and coaxially aligned to optimize the sensitivity of the system. Similar, data acquisition and motor control software were used synchronize, scanning, data acquisition, laser firing events. All data was recorded with the same data acquisition card and PC as the AR-PAM.

\subsection{Human imaging}

Before imaging, a box of the desired size was drawn on the skin of the volunteer in the region to be imaged. The hand or arm was mounted on a homemade arm holder. The volunteer was strapped into the holder to reduce motion artifacts. A homemade armrest for the volunteer's upper arm was mounted to the optical table, making the volunteer more comfortable, which also contributed to reduction in motion artifacts. The hand or arm was then placed under the membrane of the water tank for each respective PAM system. The position of the marker was determined by scanning over various positions of a large region of the skin. This process required several minutes to complete. Once oriented, the scanning range was then properly set to image the desired area. For palm images, a $5 \times 5 \mathrm{~mm}^{2}$ area was scanned. For forearm images an area of $4 \times 4 \mathrm{~mm}^{2}$ was scanned. All images were acquired within $\sim 15$ minutes, which depended on the scan range and step size.

\subsection{Image processing}

Collected data was processed with MATLAB. First, maximum amplitude projections (MAPs) of the three dimensional images were created. For AR-PAM images and the forearm image from the OR-PAM, surface signals were excluded from the construction of the MAPs. Specifically, the strong stratum corneum signal in the OR-PAM forearm image was removed during image processing. This was not necessary for the OR-PAM palm image because the epidermal layer is much thicker, causing the upper layer, the stratum corneum, to be located outside of the focal zone of the optical illumination. Hence the laser fluence on the palmer stratum corneum was sufficiently low, which generated PA signals much lower than the in-focus capillary signal beneath it. For the AR-PAM images, the entire epidermis and the capillary bed directly below it were removed during image processing. The capillary bed was excluded because these vessels are much smaller than the resolution of the system, which yield a diffuse signal and prevents higher image quality of the deeper vessels when display in an MAP. After construction, the MAPs were co-registered using standard MATLAB coregistration tools and landmarks provided by the black box of ink in each of the images. Images for different systems were plotted in different colors, AR-PAM images in green and OR-PAM images in red. The co-registered MAPs were then overlaid and displayed in a singular image. 


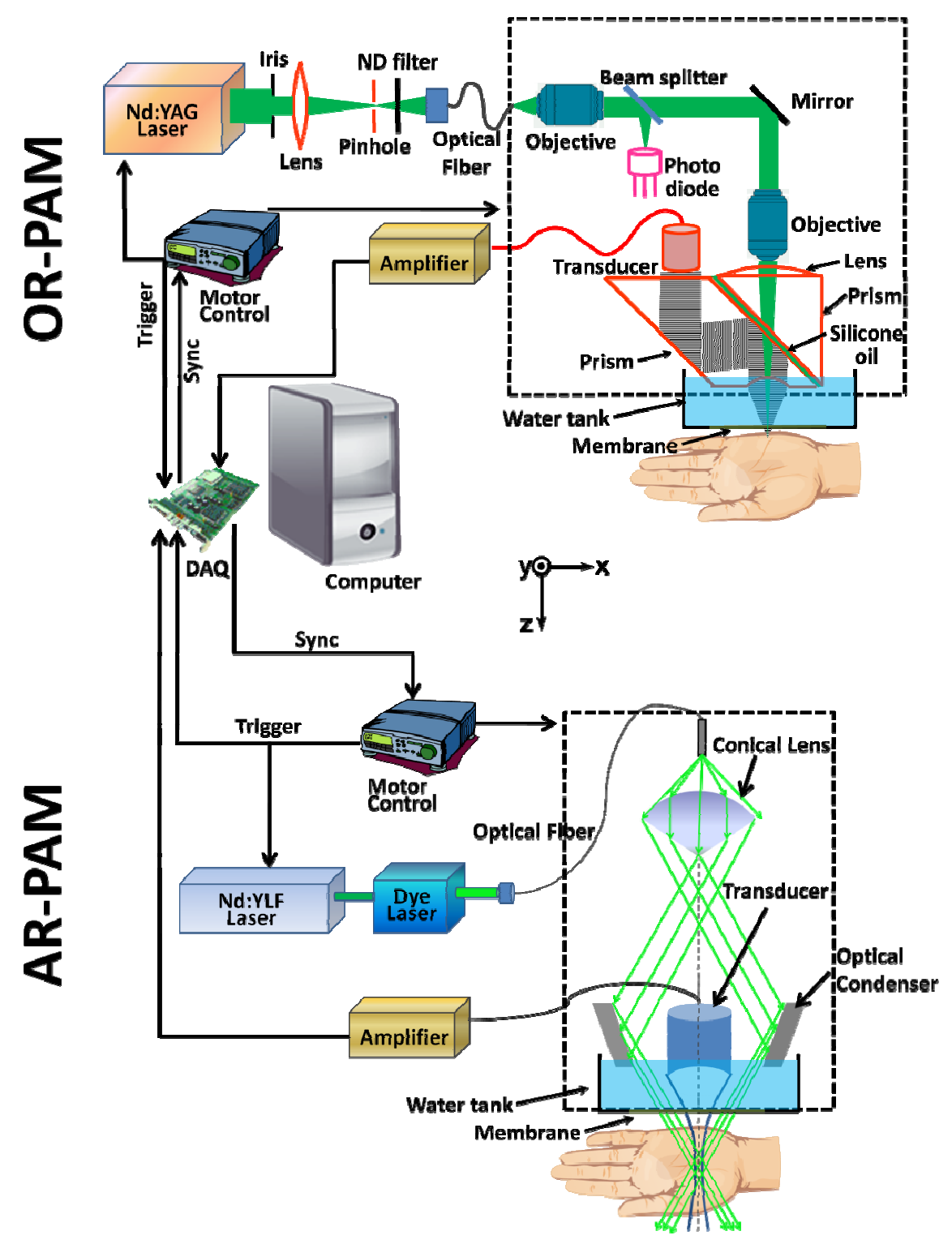

Figure 1. Schematic of both the AR-PAM and OR-PAM. Both systems shared the same data acquisition hardware and software. Each possessed its own motor control hardware, but utilized the same software. Specific of the optical deliver and ultrasound detection set-ups are shown in the schematic.

\section{RESULTS}

\subsection{Palm}

Figure 2 shows images collected from the palm of the volunteer, including a photograph of the imaged area, representative cross-sectional images (B-scans) from the 3D datasets, and MAPs from both systems. From the AR-PAM B-scan (Fig. 2b), several notable features are identifiable. Near the top of the images is the stratum corneum, the top layer of the epidermis. Immediately below it are the epidermal-dermal junctions and capillary bed. Deeper yet, is the sub-papillary plexus comprised of larger arterioles and venules. These vessels are clearly shown in the MAP image (Fig. 2e). With the OR-PAM, different structures were resolved and are identifiable. Namely, smaller artioles and venules as well as capillaries are seen in the OR-PAM images (Fig. 2(c-d)). As noted above, since the epidermis of the palm is so thick (several hundred microns), the stratum corneum layer is sufficiently far enough away from the targeted vessels that 
it was outside the focal zone of the laser focus and thus does not present strong photoacoustic signals. The overlaid MAP image in Figure 3 clearly displays the complementary structures imaged by both systems.

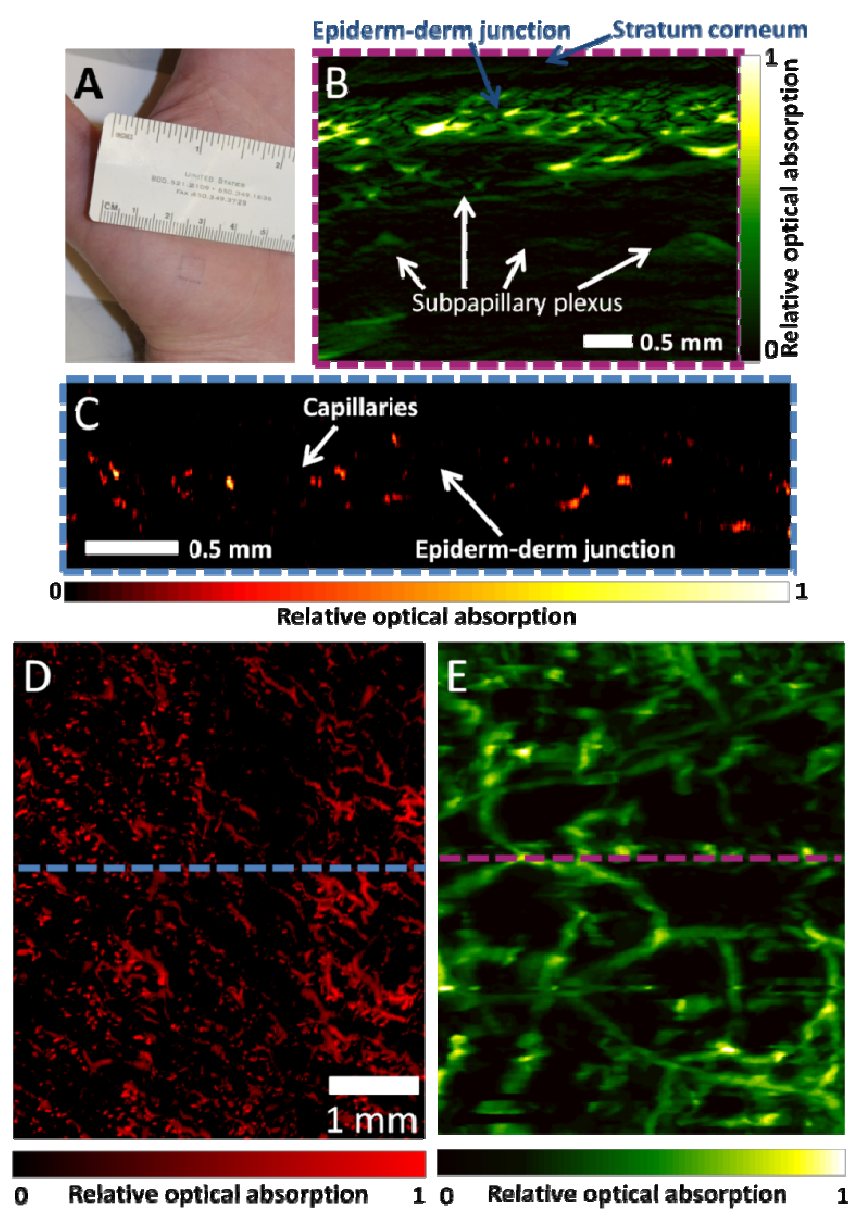

Figure 2. Images taken from the palm of a volunteer. (a) Photo indicating the imaged area. (B) Cross-section or Bscan from the AR-PAM dataset. Its position in marked by the dashed line in (E) (C) B-scan from the OR-PAM dataset. Its position marked by the dashed line in (D). (D) Maximum amplitude projection image of the 3D ORPAM data. This image has been rotated $90^{\circ}$ relative to the photo in (A). (E) Maximum amplitude projection of the 3D AR-PAM dataset. During the construction of the image, only signals below the epidermal-dermal junction and capillary bed were included. This image has been rotated $90^{\circ}$ relative to the photo in (A).

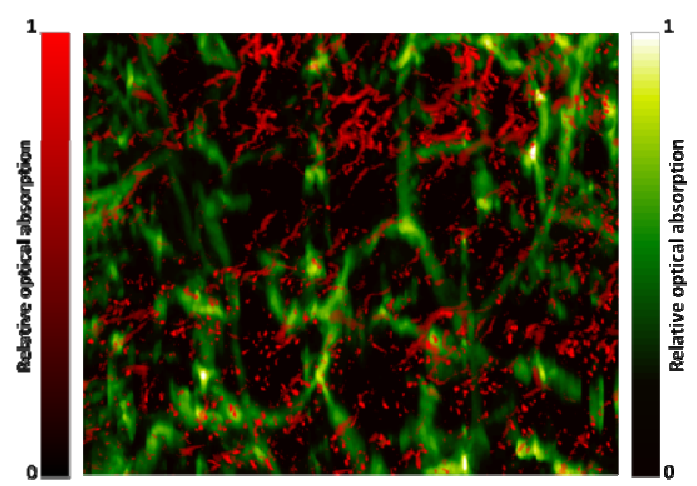

Figure 3. Overlay of the co-registered maximum amplitude projection images from palm AR-PAM and OR-PAM data show in Figure 2. The green pixels represent deeper blood vessels imaged with the AR-PAM. The red pixels represent the shallow, smaller microvessels and capillaries imaged with the OR-PAM. 


\subsection{Forearm}

Figure 4 shows images collected from the forearm of the volunteer, including a photograph of the imaged area, representative B-scans from the 3D datasets, and MAPs from both systems. For imaging skin from the forearm, the ARPAM effectively images deeper vessels, beyond $1 \mathrm{~mm}$ deep (Figure 4b). However, the epidermis is too thin and the signal from the stratum corneum is too weak for it to be distinguished. Further, capillaries and the smallest, shallowest arterioles and venules are detected but not resolved. In contrast, with the OR-PAM, the epidermal-dermal junction is clearly resolved, as are capillaries. OR-PAM can also resolve the undulations of the epidermal-dermal junction, referred to as dermal papillae. Standard skin structural models suggest that each of the papillae posses one capillary loop, which provides nutrients to cells of the epidermis. In the B-scan image for the OR-PAM, two perfused capillary vessels are clearly shown to singularly occupy individual dermal papillae (Fig. 4c). Note that the stratum corneum in forearm is much thinner than in palm and the optical fluence is still sufficient enough to generate detectable signals. A composite image of the co-registered MAPs is shown in Figure 6.
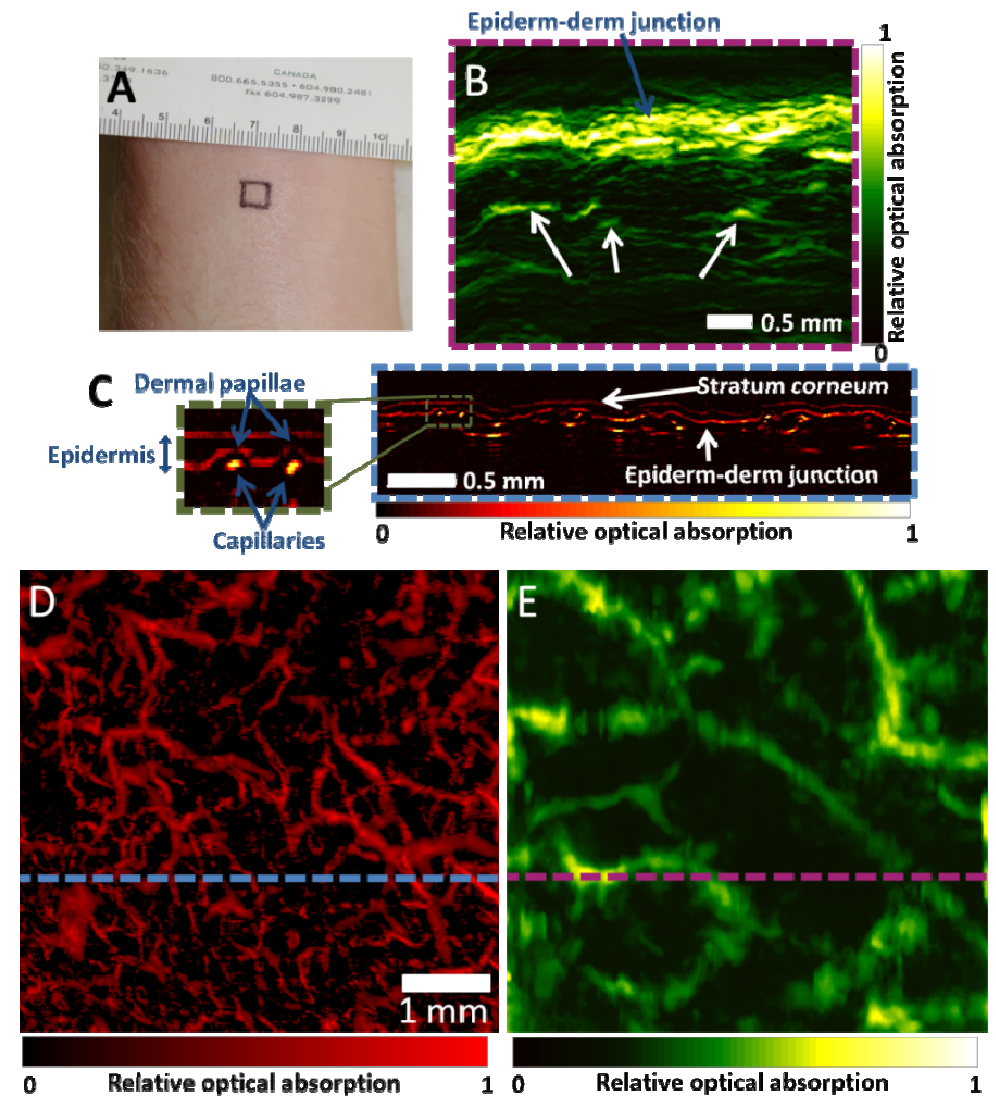

Figure 4. Images taken from the forearm of a volunteer. (a) Photo indicating the imaged area. (B) Cross-section or B-scan from the AR-PAM dataset. Its position in marked by the dashed line in (E) (C) B-scan from the OR-PAM dataset. Its position marked by the dashed line in (D). Also shown are two perfused capillaries, each located in individual papillary bumps, as is the commonly understood skin structure. (D) Maximum amplitude projection image of the 3D OR-PAM data. This image has been rotated $90^{\circ}$ relative to the photo in (A). (E) Maximum amplitude projection of the 3D AR-PAM dataset. During the construction of the image, only signals below the epidermal-dermal junction and capillary bed were included. This image has been rotated $90^{\circ}$ relative to the photo in (A). 


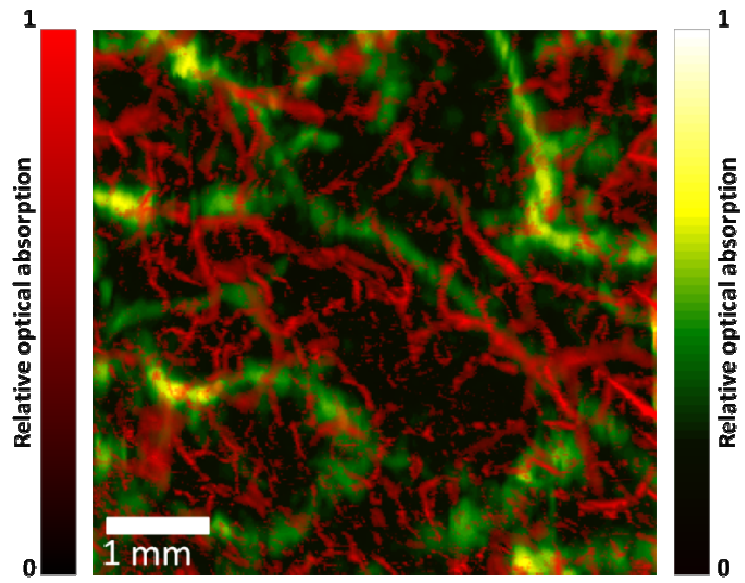

Figure 5. Overlay of the co-registered maximum amplitude projection images from forearm AR-PAM and OR-PAM data show in Figure 4. The green pixels represent deeper blood vessels imaged with the AR-PAM. The red pixels represent the shallow, smaller microvessels and capillaries imaged with the OR-PAM.

\section{SUMMARY}

The presented results demonstrate a key feature of PAM, scalability. Human skin, with its layered vascular structure whose dimensions scale with depth, provides an ideal imaging target to illustrate this advantage. From the OR-PAM and AR-PAM images, different structures in the skin were clearly resolved. Further PAM's scalability indicates that is wellsuited to more fully characterize cutaneous circulation in vivo. This capability could have impact and foster applications in circulatory and dermatological applications.

\section{ACKNOWLEDGEMENTS}

This research was supported by National Institutes of Health grants R01 EB000712, R01 EB010049, R01 CA134539, R01 EB008085, R01 CA134539, U54 CA136398, and 5P60 DK02057933. LW acknowledges financial interest in Endra Inc., which, however, did not support this research.

\section{REFERENCES}

[1] K. Maslov, H. F. Zhang, S. Hu et al., "Optical-resolution photoacoustic microscopy for in vivo imaging of single capillaries," Opt Lett, 33(9), 929-31 (2008).

[2] T. N. Erpelding, C. Kim, M. Pramanik et al., "Sentinel lymph nodes in the rat: noninvasive photoacoustic and US imaging with a clinical US system,” Radiology, 256(1), 102-10 (2010).

[3] I. M. Braverman, "The cutaneous microcirculation," J Investig Dermatol Symp Proc, 5(1), 3-9 (2000).

[4] G. Cevc, and U. Vierl, "Spatial distribution of cutaneous microvasculature and local drug clearance after drug application on the skin," J Control Release, 118(1), 18-26 (2007).

[5] C. J. Abularrage, A. N. Sidawy, G. Aidinian et al., "Evaluation of the microcirculation in vascular disease," J Vasc Surg, 42(3), 574-81 (2005).

[6] G. Micali, F. Lacarrubba, M. L. Musumeci et al., "Cutaneous vascular patterns in psoriasis," Int J Dermatol, 49(3), 249-56 (2010).

[7] K. Maslov, G. Stoica, and L. V. Wang, "In vivo dark-field reflection-mode photoacoustic microscopy," Opt Lett, 30(6), 625-7 (2005).

[8] H. F. Zhang, K. Maslov, G. Stoica et al., "Functional photoacoustic microscopy for high-resolution and noninvasive in vivo imaging," Nat Biotechnol, 24(7), 848-51 (2006). 